\title{
COMPARISON OF PRIMERS FOR RAPD-PCR FROM ENVIRONMENTAL ISOLATES OF CRYPTOCOCCUS NEOFORMANS, CRYPTOCOCCUS ALBIDUS AND CRYPTOCOCCUS LAURENTII COMPLEX
}

\section{Reginaldo dos Santos Pedroso ${ }^{1^{*}}$, Joseane Cristina Ferreira ${ }^{2}$, Karen Regina Carim da Costa ${ }^{3}$, Regina Celia Candido ${ }^{2}$}

${ }^{1}$ Escola Técnica de Saúde, Universidade Federal de Uberlândia, Uberlândia, MG, Brasil; ${ }^{2}$ Faculdade de Ciências Farmacêuticas de Ribeirão Preto, Universidade de São Paulo, Ribeirão Preto, SP, Brasil; ${ }^{3}$ Faculdade de Ciências Farmacêuticas, Universidade Federal do Amazonas, Manaus, AM, Brasil.

Submitted: June 07, 2011; Returned to authors for corrections: November 08, 2011; Approved: June 07, 2012.

\begin{abstract}
Various organisms have been characterized by molecular methods, including fungi of the genus Cryptococcus. The purposes of this study were: to determine the discriminatory potential of the RAPD (Random Amplified Polymorphic DNA) primers, the pattern of similarity of the Cryptococcus species, and discuss their useful application in epidemiological studies. We analyzed 10 isolates of each specie/group: $C$. albidus, C. laurentii complex, C. neoformans var. grubii, all from environmental source, and two ATCC strains, C. neoformans var. grubii ATCC 90112, and C. neoformans var. neoformans ATCC 28957 by RAPD-PCR using the primers CAV1, CAV2, ZAP19, ZAP20, OPB11 and SEQ6. The primers showed a good discriminatory power, revealing important differences between them and between species; the SEQ6 primer discriminated a larger number of isolates of three species. Isolates of $C$. laurentii showed greater genetic diversity than other species revealed by all six primers. Isolates of $C$. neoformans were more homogeneous. Only the primer CAV2 showed no amplification of DNA bands for C. albidus. It was concluded that the use of limited number of carefully selected primers allowed the discrimination of different isolates, and some primers (e.g., CAV2 for C. albidus) may not to be applied to some species.
\end{abstract}

Key words: Cryptococcus albidus; Cryptococcus laurentii; RAPD; Molecular Markers.

\section{INTRODUCTION}

Fungi of the genus Cryptococcus are important agents of infections in immunocompromised individuals, especially those with AIDS. C. neoformans and C. gattii are the main species involved in cryptococcosis $(9,16)$, but other species, as C. laurentii, C. albidus, C. uniguttulatus, C. luteolus, C. adeliensis have been encountered in human and animal infections in recent decades (15).

Typing of Cryptococcus isolates, especially $C$. neoformans complex (wich includes $C$. neoformans and $C$. gattii), relies on well established phenotypic characteristics, such as exoenzyme production, serotyping, morphotyping, antifungal susceptibility, killer toxin sensitivity patterns,

*Corresponding Author. Mailing address: Curso Técnico em Análises Clínicas, Escola Técnica de Saúde, Universidade Federal de Uberlândia, Avenida Amazonas, s/n, Bairro Umuarama, Uberlândia, Minas Gerais, 38400-902, Brazil.; Tel.: 55 34 3218-2218; Fax: 55 34 3218-2410.; E-mail: rpedroso@estes.ufu.br 
among other $(5,6,13)$. However, more sensitive and specific tools, as molecular typing methods, that are able to distinguish subpopulations of the same species of organisms have been developed in recent years. These tools have allowed distinguishing isolates with different profiles of virulence factors, susceptibility to antifungal drugs, and to discriminate strains from distinct geographic areas $(12,28)$.

Several molecular typing methods, as reliable and practical options, have been studied and improved, although not all methods are equally discriminatory. Randomly amplified polymorphic DNA (RAPD), pulsed-field gel electrophoresis (PFGE), restriction fragment length polymorphism (RFLP), amplified fragment length polymorphism (AFLP), and more recently, multigene sequence analysis to multilocus sequence typing (MLST) are the most commonly used for the $C$. neoformans complex analysis. Actually, MLST has been purposed as the standardized method for strain typing of $C$. neoformans isolates $(17,21)$. On the other hand, the RAPD has been one of the most used for typing different microorganisms, such as Candida albicans (3, 22). These markers have been applied in detecting polymorphisms, identification and isolation of specific DNA fragments, and have application in genomic analysis of a wide variety of species, with a relatively low cost (27). However, a good typability and accuracy of the method is desirable, so it is important to choose primers that allow a high discriminatory power of the isolates.

The purposes of the present study were to determine the discriminatory potential of the RAPD primers, to analyze the molecular profile of three species of Cryptococcus by RAPDPCR, and to discuss their possible usefulness in epidemiological studies.

\section{MATERIALS AND METHODS}

\section{Microorganisms}

Ten isolates of each specie/group, C. albidus (identified as
CRA01, CRA03, CRA04, CRA06, CRA07, CRA08, CRA10, CRA11, CRA12 and CRA15), C. laurentii complex (CRL01, CRL02, CRL04, CRL05, CRL08, CRL09, CRL10, CRL11, CRL12 and CRL13) and C. neoformans var. grubii (CN13, CN17, CN18, CN19, CN20, CN22, CN23, CN24, CN25, CN26), were obtained from air and avian droppings in public urban areas inhabited by pigeons at the city of Ribeirão Preto, State of São Paulo, Brazil (18). Identification of the isolates was done by classical methods and confirmed by ID32C system (Bio-Merieux, Marcy I'Etoile, France). C. neoformans var. grubii ATCC 90112 and C. neoformans var. neoformans ATCC 28957 were included in this study. The strains were stored in both at $-20^{\circ} \mathrm{C}$ in $\mathrm{BHI}-$ glycerol and at $22-28^{\circ} \mathrm{C}$ in Sabouraud dextrose agar (SDA) subcultured bimonthly.

\section{Genomic DNA extraction and RAPD-PCR}

Genomic DNA was extracted as described by Bolano et al. (7), with some modifications (19) and stored at $-20^{\circ} \mathrm{C}$. The reactions were performed using the primers described in Table 1 , in a final volume of $25 \mu \mathrm{L}$, containing $2.5 \mu \mathrm{L}$ of $10 \mathrm{x}$ enzyme buffer with $\mathrm{KCl}$ (Fermentas, Glen Burnie, MD, USA), 3 $\mathrm{mmol} / \mathrm{L}$ of $\mathrm{MgCl}_{2}, 0.2 \mathrm{mmol} / \mathrm{L}$ of dNTPs, $50 \mathrm{ng}$ of DNA, 0.4 $\mathrm{mmol} / \mathrm{L}$ of each primer and $1 \mathrm{U}$ of Taq DNA polymerase (Fermentas, Glen Burnie, MD, USA). PCR was performed in a Mastercycler thermocycler (Eppendorf, Hamburg, Germany). The reactions were as follows: initial denaturation at $94^{\circ} \mathrm{C}$ for 5 $\min , 40$ cycles of $1 \mathrm{~min}$ at $94^{\circ} \mathrm{C}, 1 \mathrm{~min}$ at $36^{\circ} \mathrm{C}, 2 \mathrm{~min}$ at $72^{\circ} \mathrm{C}$, followed by final extension for $10 \mathrm{~min}$ at $72^{\circ} \mathrm{C}$. Control tubes without genomic DNA were included in each reaction as a control. Amplification products were submitted electrophoresis on $1 \%$ agarose gel in TBE buffer $0.5 \times(89 \mathrm{mmol} / \mathrm{L}$ of Trisborate and $2 \mathrm{mmol} / \mathrm{L}$ of EDTA), at $80 \mathrm{~V}$ for $3 \mathrm{~h}$.

The processed gels were stained with $1 \mathrm{mg} / \mathrm{L}$ of ethidium bromide (Invitrogen, São Paulo, SP, Brazil), visualized with UV transilluminator and the images captured by a capture system (Alpha-Innotech, San Leandro, CA, USA). Molecular size standards Lambda-HindIII (Fermentas, Glen Burnie, MD, 
USA) were used as reference to band sizes. Only intense and reproducible fragments were considered for analysis.
Reproducibility was assessed by PCR-amplification on two different occasions.

Table 1. Primer sequences for RAPD-PCR.

\begin{tabular}{ccc}
\hline Primer & \multicolumn{1}{c}{ Sequence } & References \\
\hline CAV1 & 5'-CCC GTC AGC A-3' & 1,10 \\
CAV2 & 5'-AAC GCG CAA C-3' & 10 \\
ZAP19 & 5'-AAG AGC CCG T-3' & 4,8 \\
ZAP20 & 5'-GCG ATC CCC A-3' & 4,8 \\
OPB11 & 5'-GTAGACCCGT-3' & 14 \\
SEQ6 & 5'-CCCGTCAGCA-3' & 19 \\
\hline
\end{tabular}

The Jaccard coefficient $\left(\mathrm{S}_{j}\right)$ was used to calculate the distance between the isolates according to comparison of the bands and their sizes (3). The Multi-variate Statistical Package 3.0 (MVSP 3.0, Kovach Computing Services, Pentraeth, Wales, UK) software was used to analyze the differences. A dendrogram was generated from the data obtained by the cluster method UPGMA (Unweighted Pair Group Method using Arithmetic Averages). $\mathrm{A} \mathrm{S}_{j}$ value of 1.00 indicated that the isolates were identical; values between 0.81 and 0.99 represented high similarity, but not identity, and suggest the occurrence of microevolution on a given isolate; values below 0.80 represented unrelated isolates $(3,22)$.

\section{RESULTS}

RAPD patterns for isolates in this study have been translated into the dendrograms (Figures 1 to 3 ).

C. laurentii complex isolates had the greatest genetic diversity. Identical patterns $\left(\mathrm{S}_{j}=1.00\right)$ were found only with primer CAV1 in a few isolates; three isolates (CRL01, CRL02 and CRL12) constituted one cluster and two isolates (CRL10 and CRL11) another one. All isolates were separated from each other by some other primers, that separated all isolates with similarities ranging from 0.00 to 0.800 . The primer OPB11 showed that CRL09 is not related with any other isolates. Figure 1 shows the dendrogram of $C$. laurentii complex isolates.

C. albidus isolates, in general, showed higher genetic similarity than $C$. laurentii isolates. Only the primer CAV2 did not amplify any DNA bands. Primers ZAP19 and CAV1 formed similar clusters, that had at least seven identical isolates; six groups $\left(\mathrm{S}_{j}=0.135\right.$ to 0.800$)$ were clustered by SEQ6, four of them with a pair of identical isolates $\left(\mathrm{S}_{j}=1.00\right)$. OPB11 grouped six identical isolates (CRA01, CRA06, CRA07, CRA08, CRA10 and CRA11) highly related to two other isolates (CRA03 with $\mathrm{S}_{j}=0.842$ and CRA12, $\left.\mathrm{S}_{j}=0.875\right)$.

C. neoformans isolates, including ATCC strains, formed three unrelated cluster $(\mathrm{Sj} \leq 0.80)$, with the primers CAV1, ZAP20 and OPB11, all of them grouping the same isolates, distributed as follows: one cluster with eight identical isolates, the other with three isolates and another one composed by 28957. Primer CAV2 formed two cluster, one formed by 28957 (var. neoformans) and the other grouping all identical isolates (all of var. grubbi). The primers ZAP19 and SEQ6 (see Figure 4) had higher discriminatory power between the primers tested, forming six and eight groups, respectively. 

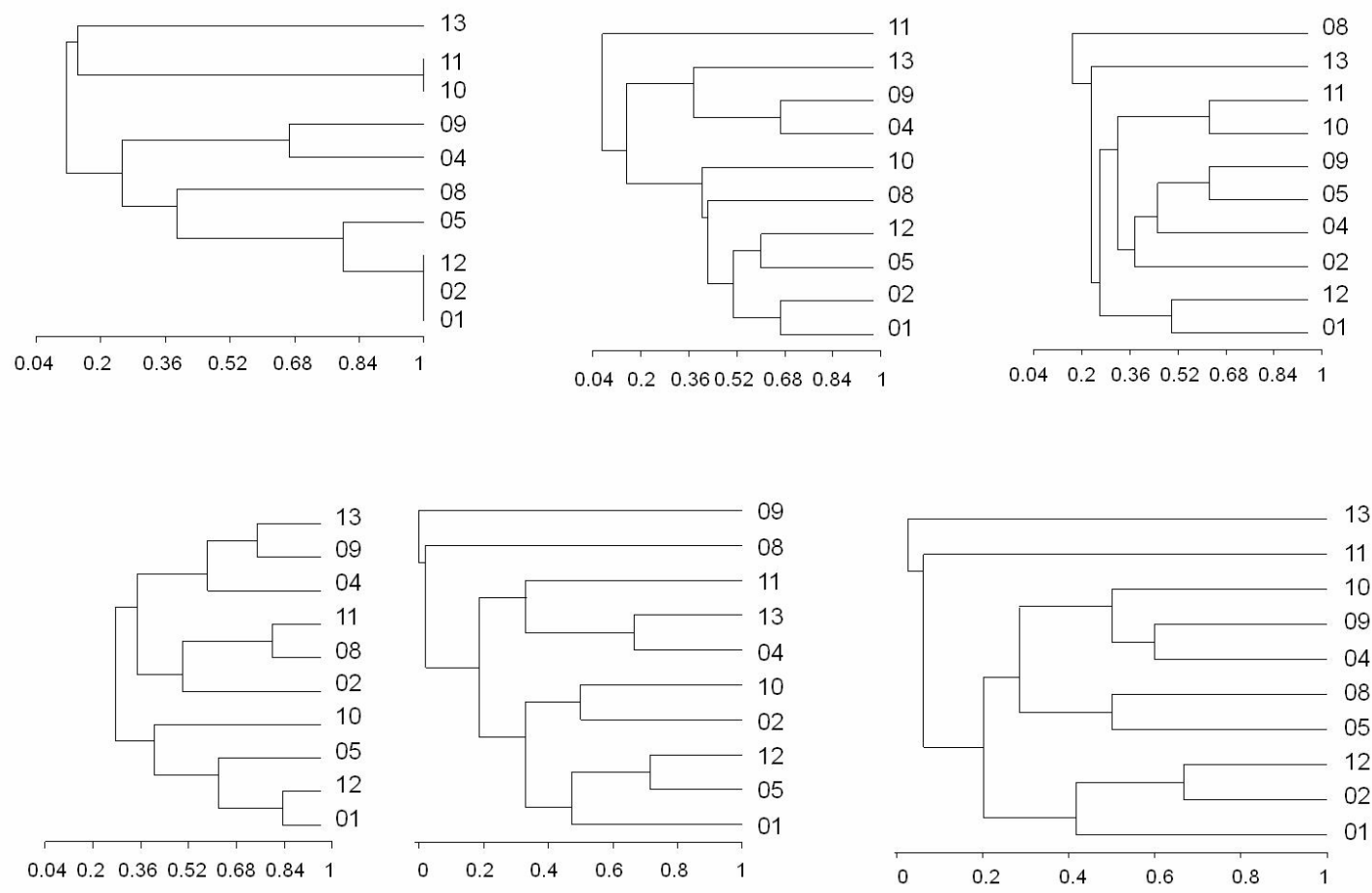

Figure 1. Dendrograms showing the relationships of $C$. laurentii isolates generated from the RAPD-PCR profiles obtained with primers (from left to right) CAV1, CAV2 and ZAP19 (above); ZAP20, OPB11 and SEQ6 (below).
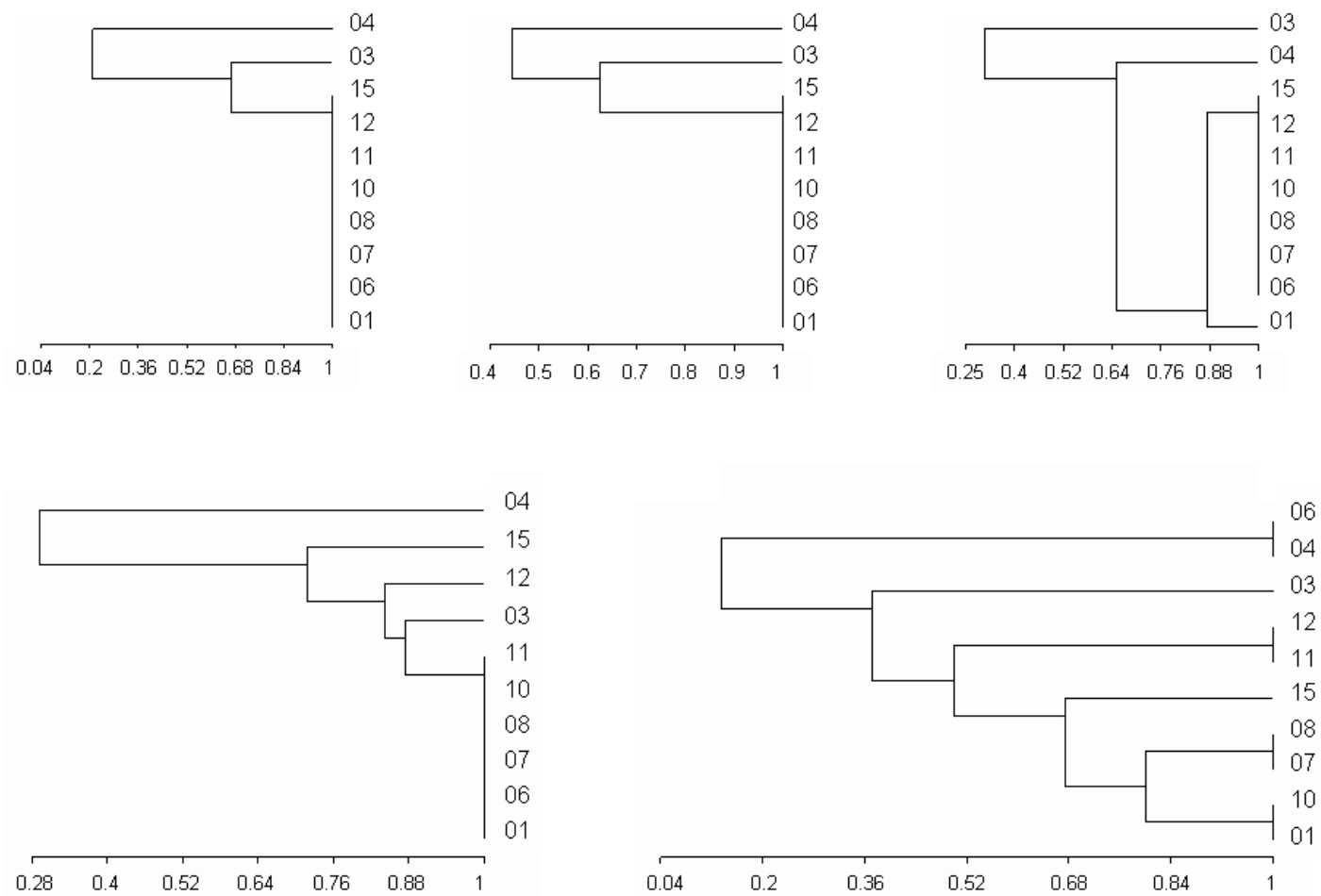

Figure 2. Dendrograms showing the relationships of $C$. albidus isolates generated from the RAPD-PCR profiles obtained with primers (from left to right) CAV1, ZAP19 and ZAP20 (above); OPB11 and SEQ6 (below). 

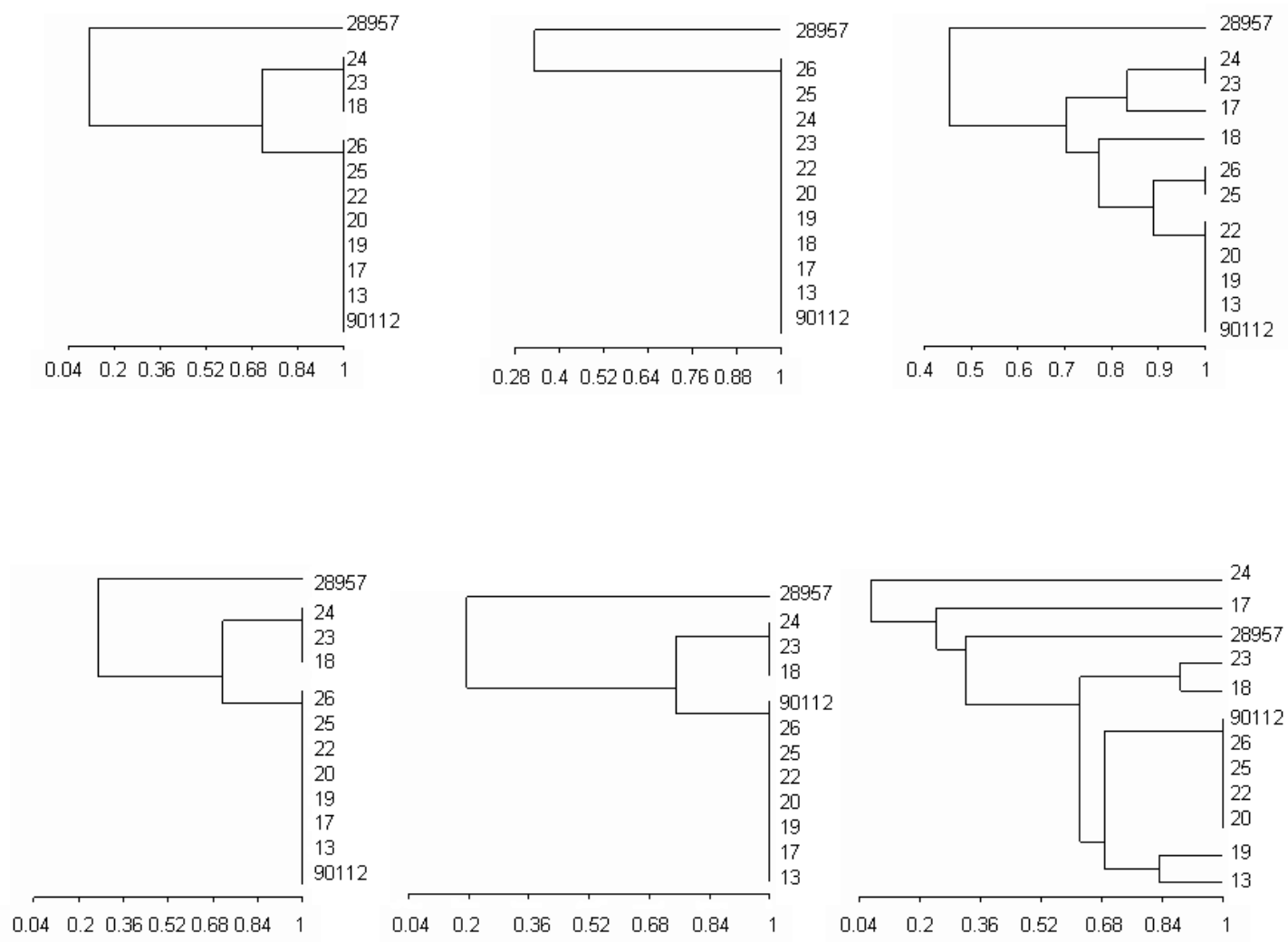

Figure 3. Dendrograms showing the relationships of $C$. neoformans isolates generated from the RAPD-PCR profiles obtained with primers (from left to right) CAV1, CAV2, ZAP19 (above); ZAP20, OPB11 and SEQ6 (below).

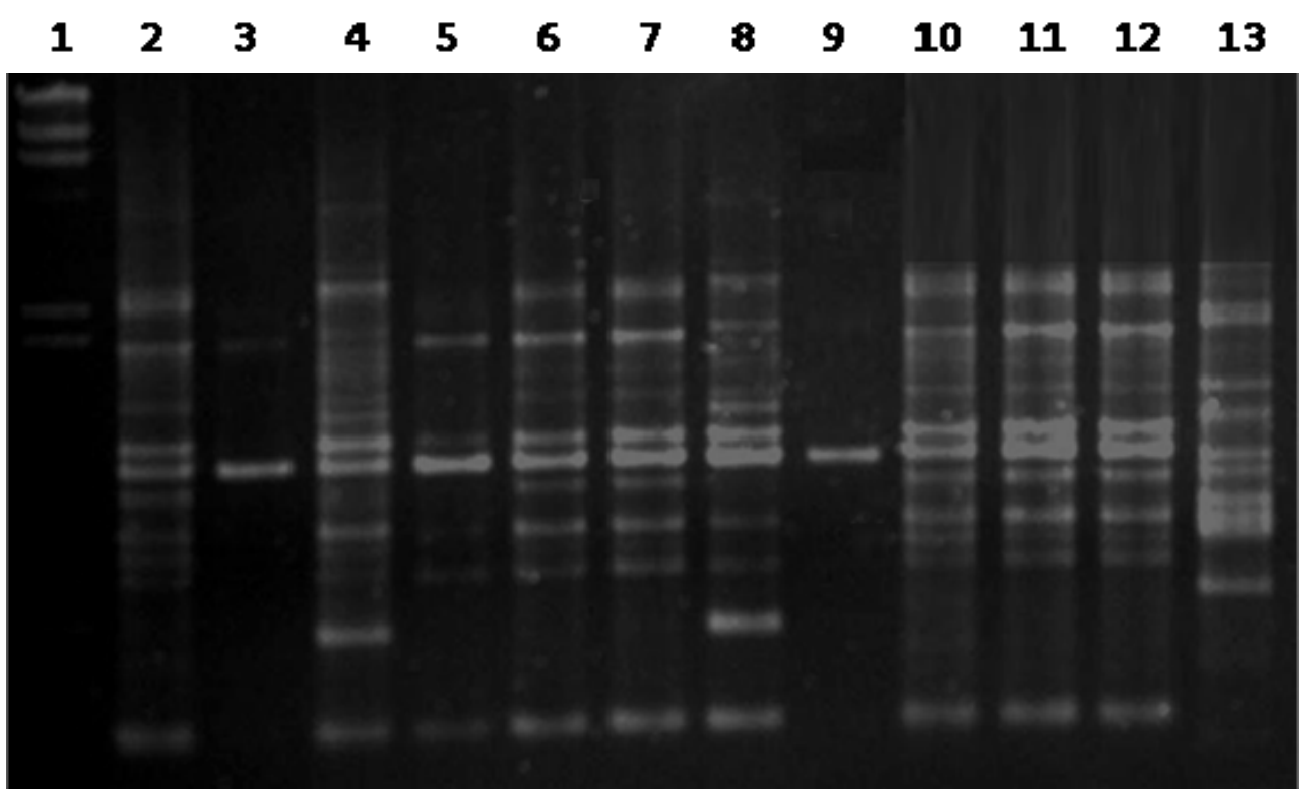

Figure 4. Examples of amplification obtained with the primer SEQ6. Line 1: molecular size marker (Lambda DNA/Hind III; Fermentas); lines 2 to 13 represent $C$. neoformans isolates 13, 17, 18, 19, 20, 22, 23, 24, 25, 26, ATCC 90112 and ATCC 28957, respectively. 


\section{DISCUSSION}

Molecular techniques have been widely used to study genetic variations in uni- and multicellular organisms $(1-4,28)$, and RAPD-PCR has been used in studies of human pathogenic fungi as well as those causing diseases in animals and plants (1, 28). Molecular studies of microorganisms contribute to the epidemiological studies and are also useful in the relationship between molecular profile and phenotypic characteristics (12).

In this study, we observed high genetic variability among isolates of $C$. laurentii complex with all the primers used, as demonstrated in previous studies $(19,23,25)$. The greatest differences among isolates of $C$. laurentii complex (minor $\mathrm{S}_{j}$ ) were observed with the primers CAV2, ZAP19, OPB11 and SEQ6. That heterogeneity is probably caused by different species and subspecies that constitute the $C$. laurentii complex, which can only be identified by more specific methods, such as rDNA sequencing (D1/D2 and/or ITS regions).

In clinical or medical laboratory, especially in Brazil, the laboratories are able to identify the $C$. laurentii complex, but not the accurate specie or subspecie. In this line, the use of molecular methods such as RAPD-PCR associated with the phenotypic tests can help identify the species or subspecies of the microorganisms and also their typing.

C. albidus is another specie that is reported as a species complex. In some isolates have been observed a high genetic heterogeneity, revealed by different molecular methods (1, 19, 24). However, in this study we demonstrated that most isolates had high similarity or were identicals (Figure 2). Among the primer used, we observed that CAV2 did not amplify any bands. Despite the small number of $C$. albidus isolates studied, the CAV2 primer seems inappropriate and ZAP20, OPB11 and SEQ6 were the primers that formed more clusters and are most promising for use in studies envolving C. albidus.

Isolates of $C$. neoformans were best discriminated by primer SEQ6, that formed eight groups, one of them grouping five isolates, including strain ATCC 90112, a strain that belongs to the var. grubii isolated from the cerebrospinal fluid in the United States. The identity revealed by most isolates of C. neoformans suggests that new primers should be researched to increase the discriminatory power. Among the primers utilized, we noted that C. neoformans ATCC 28957, a strain of the var. neoformans, formed an evident separate group with all the primers. It is interesting to note that the primer CAV2 grouped all isolates except 28957, as shown in Figure 3.

The study of molecular markers is promising for the typing of microorganisms. These studies are interesting to study the relationship between molecular profile and phenotypic characteristics, such as in vitro virulence factors production (laccases, phospholipases, proteases), phenotypic switching, resistance to antifungal drugs, or associations between geographic origin of the isolates and molecular profiles. Certain molecular profiles may be related to specific features of the phenotype, as has been shown for $C$. neoformans and another species isolates from different parts of the world $(1,3,4,10,14,26)$. Recently the evidence of the relationship between susceptibility to antifungal agents and different genotypes of $C$. neoformans/ C. gattii complex has increased (26).

The use of a single molecular marker is usually not sufficient to discriminate a large number of isolates. The establishment of a rational screening protocol, with primers carefully chosen and carried out in sequential reactions, or even the sequential analysis of results can allow discrimination of isolates or even increase the discriminatory power of the method, expanding the field of application of molecular markers. More studies with larger numbers of isolates and primers, available for practical application in clinical and research laboratories, will facilitate the work of researchers, will reduce cost and time consuming.

In conclusion, we observed that the use of limited number of carefully selected primers allowed the discrimination of different isolates, and not every primers (e.g., CAV2 for $C$. albidus) can be applied to any species. Future studies involving 
C. laurentii complex will be required to detail the identification at the level of the species or subspecies, for example, by comparison with a validated method such as rDNA sequencing (D1/D2 domain or ITS). More studies, however, are necessary to determine the effectiveness of this tool and primers studied here and other in clinical isolates, as well as in veterinary and environmental isolates.

\section{ACKNOWLEDGEMENTS}

This study was supported in part by CNPq (Conselho Nacional de Desenvolvimento Científico e Tecnológico), Brazil.

\section{REFERENCES}

1. Almeida, A.M.F.; Matsumoto, M.T.; Baeza, L.C.; Oliveira e Silva, R.B.; Kleiner, A.A.; Melhem, M.D.E.S.; Mendes-Giannini, M.J.; Laboratory Group on Cryptococcosis. (2007). Molecular typing and antifungal susceptibility of clinical sequential isolates of Cryptococcus neoformans from São Paulo State, Brazil. FEMS Yeast Res. 7 (1), 152-164.

2. Arif, I.A.; Bakir, M.A.; Khan, H.A.; Al Farhan, A.H.; Al Homaidan, A.A.; Bahkali, A.L.; Al Sadoon, M.; Shobrak, M. (2010). A brief review of molecular techniques to assess plant diversity. Int. J. Mol. Sci. 11 (5), 2079-2096.

3. Bacelo, K.L.; Costa, K.R.C.; Ferreira, J.C.; Candido, R.C. (2010). Biotype stability of Candida albicans isolates after culture storage determined by randomly amplified polymorphic DNA and phenotypical methods. Mycoses. 53 (6), 468-474.

4. Blasi, E.; Brozzetti, A.; Francisci, D.; Neglia, R.; Cardinali, G.; Bistoni, F.; Vidotto, V.; Baldelli, F. (2001). Evidence of microevolution in a clinical case of recurrent Cryptococcus neoformans meningoencephalitis. Eur. J. Clin. Microbiol. Infect. Dis. 20 (8), 535-543.

5. Boekhout, T.; Belkun, A.; Leenders, A.C.A.; Verbrugh, H.A.; Mukamurangwa, P.; Swinne, D.; Scheffers, W.A. (1997). Molecular typing of Cryptococcus neoformans: taxonomic and epidemiological aspects. Int. J. Syst. Bacteriol. 47 (2), 432-442.

6. Boekhout, T.; Scorzetti, G. (1997). Differential killer toxin sensitivity patterns of varieties of Cryptococcus neoformans. J. Med. Vet. Mycol. 35 (2), 147-149.

7. Bolano, A.; Stinchi, S.; Preziosi, R.; Bistoni, F.; Allegrucci, M.; Baldelli, F.; Martini, A.; Cardinali, G. (2001). Rapid methods to extract DNA and RNA from Cryptococcus neoformans. FEMS Yeast Res. 1 (3), 221-224.
8. Cardinali, G.; Martini, A.; Preziosi, R.; Bistoni, F.; Baldelli; F. (2002). Multicenter comparison of three different analytical systems for evaluation of DNA banding patterns from Cryptococcus neoformans. J. Clin. Microbiol. 40 (6), 2095-2100.

9. Casadevall, A.; Perfect, J.R. (1998). Cryptococcus neoformans. ASM Press, Washington, D.C.

10. Cavalcante, S.C.; Freitas, R.S.; Vidal, M.S.; Dantas, K.C.; Levi, J.E.; Martins, J.E. (2007). Evaluation of phenotypic and genotypic alterations induced by long periods of subculturing of Cryptococcus neoformans strains. Mem. Inst. Oswaldo Cruz. 102 (1), 11-17.

11. Fonseca, A.; Scorzetti, G.; Fell, J.W. (2000). Diversity in the yeast Cryptococcus albidus and related species as revealed by ribosomal DNA sequence analysis. Can. J. Microbiol. 46 (1), 7-24.

12. Foxman, B.; Riley, L. (2001). Molecular epidemiology: focus on infection. Am. J. Epidemiol. 153 (12), 1135-1141.

13. Hunter, P.R. (1991). A critical review of typing methods for Candida albicans and their applications. Crit. Rev. Microbiol. 17 (6), 417-434.

14. Jain, P.; Khan, Z.K.; Bhattacharya, E.; Ranade, S.A. (2001). Variation in random amplified polymorphic DNA (RAPD) profiles specific to fluconazole resistant and sensitive strains of Candida albicans. Diagn. Microbiol. Infect. Dis. 41 (3), 113-119.

15. Khawcharoenporn, T.; Apisarnthanarak, A.; Mundy, L.M. (2007). Nonneoformans cryptococcal infections: a systematic review. Infection. 35 (2): 51-58.

16. Kwon-Chung, K.J.; Bennett, J.E. (1992). Medical mycology. Lea \& Febiger, Philadelphia.

17. Meyer, W.; Aanensen, D.M.; Boekhout, T.; Cogliati, M.; Dias, M.R.; Esposto, M.C.; Fisher, M.; Gilgado, F; Hagen, F.; Kaocharoen, S.; Litvintseva, A.P.; Mitchell, T.G.; Simwami, S.; Trilles, L.; Viviani, M.A.; Kwon-Chung, J. (2009). Consensus multi-locus sequence typing scheme for Cryptococcus neoformans and Cryptococcus gattii. Med. Mycol. 47 (6): 561-570.

18. Pedroso, R.S.; Ferreira, J.C.; Candido, R.C. (2009). The isolation and characterization of virulence factors of Cryptococcus spp. from saprophytic sources in the city of Ribeirão Preto, São Paulo, Brazil. Microbiol. Res. 164 (2): 221-227.

19. Pedroso, R.S.; Ferreira, J.C.; Lavrador, M.A.; Maffei, C.M.; Candido, R.C. (2009). Evaluation of the experimental inoculation of Cryptococcus albidus and Cryptococcus laurentii in normal mice: virulence factors and molecular profile before and after animal passage. Mycopathologia. 168 (2): 59-72.

20. Riederer, K.M.; Ramanathan, J.; Barczak, J.; Baran, J.; Khatib, R. (2002). Utility of a pre-optimized kit for random amplified polymorphic DNA in typing Candida albicans. Can. J. Microbiol. 48 (4): 369-373.

21. Sidrim, J.J.C.; Costa, A.K.F.; Cordeiro, R.A.; Brilhante, R.S.N.; Moura, F.E.A.; Castelo-Branco, D.S.C.M.; Araújo Neto, M.P.; Rocha, M.F.G. (2010). Molecular methods for the diagnosis and characterization of 
Cryptococcus: a review. Can. J. Microbiol. 56 (6): 445-458.

22. Soll, D.R. (2000). The ins and outs of DNA fingerprinting the infections fungi. Clin. Microbiol. Rev. 13 (2): 332-370.

23. Sugita, T.; Takashima, M.; Ikeda, R.; Nakase, T.; Shinoda, T. (2000). Intraspecies diversity of Cryptococcus laurentii as revealed by sequences of internal transcribed spacer regions and 28S rRNA gene and taxonomic position of Cryptococcus laurentii clinical isolates. J. Clin. Microbiol. 38 (4): 1468-1471.

24. Sugita, T.; Takashima, M.; Ikeda, R.; Nakase, T.; Shinoda, T. (2001). Intraspecies diversity of Cryptococcus albidus isolated from humans as revealed by sequences of the internal transcribed spacer regions. Microbiol. Immunol. 45 (4): 291-297.

25. Takashima, M.; Sugita, T.; Shinoda, T.; Nakase, T. (2003). Three new combinations from the Cryptococcus laurentii complex: Cryptococcus aureus, Cryptococcus carnescens and Cryptococcus peneaus. Int. J. Syst. Evol. Microbiol. 53 (Pt 4): 1187-1194.

26. Trilles, L.; Meyer, W.; Wanke, B.; Guarro, J.; Lazera, M. (2012). Correlation of antifungal susceptibility and molecular type within the Cryptocccus neoformans/ C. gattii species complex. Med. Mycol. 50 (3): 328-332.

27. Williams, J.G.K.; Kubelik, A.R.; Livak, K.J.; Rafalski, J.A.; Tingey, S.V. (1990). DNA polymorphisms amplified by arbitrary primers are useful as genetic markers. Nucleic Acids Res. 18 (22): 6531-6535.

28. Zaidi, N.; Konstantinou, K.; Zervos, M. (2003). The role of molecular biology and nucleic acid technology in the study of human infection and epidemiology. Arch. Pathol. Lab. Med. 127 (9): 1098-1105. 